\title{
MULTI-PEAK SOLUTIONS FOR A WIDE CLASS OF SINGULAR PERTURBATION PROBLEMS
}

\author{
JUNCHENG WEI AND MATTHIAS WINTER
}

\begin{abstract}
In this paper we are concerned with a wide class of singular perturbation problems arising from such diverse fields as phase transitions, chemotaxis, pattern formation, population dynamics and chemical reaction theory. We study the corresponding elliptic equations in a bounded domain without any symmetry assumptions. We assume that the mean curvature of the boundary has $\bar{M}$ isolated, non-degenerate critical points. Then we show that for any positive integer $m \leq \bar{M}$ there exists a stationary solution with $M$ local peaks which are attained on the boundary and which lie close to these critical points. Our method is based on Liapunov-Schmidt reduction.
\end{abstract}

\section{INTRODUCTION}

In this paper we are concerned with a wide class of singular perturbation problems including the Cahn-Hilliard equation for phase transitions in metallurgy, the Keller-Segal model in chemotaxis, the Gierer-Meinhardt system in pattern formation and a famous model from population dynamics and chemical reaction theory.

Let us begin with some background and a summary of our results for the Cahn-Hilliard equation. Among the models mentioned above this is mathematically the most complicated one because of its non-local character. Then we will describe the other problems which can be dealt with by our approach.

The Cahn-Hilliard equation [6] is a commonly used macroscopic fieldtheoretical model of processes such as phase separation in a binary alloy. It

1991 Mathematics Subject Classification. Primary 35B40, 35B45; Secondary 35J40.

Key words and phrases. Phase Transition, Nonlinear Elliptic Equations. 
can be derived from a Helmholtz free energy

$$
E(u)=\int_{\Omega}\left[F(u(x))+\frac{1}{2} \epsilon^{2}|\nabla u(x)|^{2}\right] d x
$$

where $\Omega$ is the region occupied by the body, $u(x)$ is a conserved order parameter representing for example the concentration of one of the components, and $F(u)$ is the free energy density which has a double well structure at low temperatures. The prototype for the free energy density is $F(u)=\left(1-u^{2}\right)^{2}$.

The constant $\epsilon$ is proportional to the range of intermolecular forces and the gradient term is a contribution to the free energy coming from spatial fluctuations of the order parameter. Moreover the mass $\bar{m}=\frac{1}{|\Omega|} \int_{\Omega} u d x$ is a given constant. Thus a stationary solution of $E(u)$ under $\bar{m}=\frac{1}{|\Omega|} \int_{\Omega} u d x$ takes the form

$$
\left\{\begin{array}{l}
\epsilon^{2} \Delta u-f(u)=\sigma_{\epsilon} \quad \text { in } \Omega, \\
\frac{\partial u}{\partial \nu}=0 \quad \text { on } \Omega,
\end{array}\right.
$$

where $f(u)=F^{\prime}(u)$ and $\sigma_{\epsilon}$ is a constant.

There have been numerous studies of the Cahn-Hilliard equation. The global minimizer of $E(u)$ has a transition layer. More precisely there exists an open set $\Gamma \subset \Omega$ such that if $\epsilon$ is small enough $u_{\epsilon}$ is a global minimizer and $u_{\epsilon} \rightarrow 1$ on $\Omega \backslash \bar{\Gamma}, u_{\epsilon} \rightarrow-1$ on $\Gamma$ and $\partial \Gamma \cap \bar{\Omega}$. Furthermore, $\Gamma$ is a surface whose area is minimal under the mass constraint and which has constant mean curvature, see [23]. The dynamics has been studied extensively, see for example [3], [9], [10], [30]. Also local minimisers have been studied and their transition layer structure has been established in [20]. In this paper we are interested in solutions of (1.1) with spike layers. In the one dimensional case, Bates and Fife [5] studied nucleation phenomena for the Cahn-Hilliard equation and proved the existence of three monotone nondecreasing stationary solutions when $\bar{m}$ is in the metastable region $(\sqrt{1 / 3}<\bar{m}<1$ ), (a) the constant solution $u \equiv \bar{m}$, (b) a boundary spike layer solution where the layer is located at the left-hand endpoint, (c) a transition layer solution with a layer in the interior of the material. 
Motivated by the results of [5], in [34] we constructed a boundary spike layer solution to (1.1) for $\epsilon<<1$ in the higher dimensional case when $\bar{m}$ is in the metastable region.

In this paper we extend the approach to construct multi-peak solutions to the Cahn-Hilliard equation.

To our knowledge these papers are the first to establish this kind of results for the Cahn-Hilliard equation in higher dimensions without any symmetry assumptions on $\Omega$.

Naturally these stationary solutions are essential for the understanding of the dynamics of the corresponding evolution process. While Bates and Fife [5] prove some results in this direction for the one dimensional case these questions are open for higher dimensions.

In [16], [17] in the one dimensional case the number of all stationary solutions is counted by arguments using transversality. Furthermore, the energy levels of stationary solutions and their connecting orbits are established.

Before we state our main assumptions we make the following transformation.

$$
\begin{gathered}
v=\bar{m}-u, \\
g(v)=-f(\bar{m})+f(\bar{m}-v) .
\end{gathered}
$$

Rewrite

$$
g^{\prime}(0)=-m, g(v)=-m v+h(v) .
$$

Then equation (1.1) becomes

$$
\begin{cases}\epsilon^{2} \Delta v-m v+h(v)-\frac{1}{|\Omega|} \int_{\Omega} h(v)=0 & \text { in } \Omega \\ \frac{\partial v}{\partial \nu}=0 & \text { on } \partial \Omega .\end{cases}
$$

Our main result can be stated as follows.

Theorem 1.1. Let $\Omega$ be a bounded smooth domain in $R^{N}(N \geq 2)$. For $i=1,2, \ldots, M$ let $P_{0, i} \in \partial \Omega$ be such that $\nabla_{\tau P_{0, i}} H\left(P_{0, i}\right)=0$ where $H(P)$ is the mean curvature of $\partial \Omega$ at $P$ and $\nabla_{\tau P_{0, i}}$ is the tangential derivative at 
$P_{0, i}$. Furthermore, assume that $\left(\nabla_{\tau P_{0, i}} \nabla_{\tau P_{0, j}} H\left(P_{0, i}\right)\right)$ is nondegenerate for $i, j=1, \ldots, M$.

Then for $\epsilon<<1$ there exists a solution $v_{\epsilon}$ of (1.2) such that $v_{\epsilon} \rightarrow 0$ in $C_{l o c}^{1}\left(\bar{\Omega} \backslash\left\{P_{0,1}, P_{0,2}, \ldots, P_{0, M}\right\}\right), v_{\epsilon}$ has exactly $M$ local maximum points $P_{\epsilon, i}$ and $P_{\epsilon, i} \in \partial \Omega, P_{\epsilon, i} \rightarrow P_{0, i}, v_{\epsilon}\left(P_{\epsilon, i}\right) \rightarrow V(0)>0$. Moreover

$$
\epsilon^{-N}\left\{\int_{\Omega} \epsilon^{2}\left|\nabla v_{\epsilon}-\sum_{i=1}^{M} \nabla V\left(\frac{x-P_{\epsilon, i}}{\epsilon}\right)\right|^{2}+\int_{\Omega}\left|v_{\epsilon}-\sum_{i=1}^{M} V\left(\frac{x-P_{\epsilon, i}}{\epsilon}\right)\right|^{2}\right\} \rightarrow 0
$$

as $\epsilon \rightarrow 0$ where $V(y)$ is the unique solution of

$$
\left\{\begin{array}{l}
\Delta V-m V+h(V)=0, \\
V(0)=\max _{y \in R^{N}} V(y), V>0, \\
V(y) \rightarrow 0 \text { at } \infty
\end{array}\right.
$$

(By the results of [13] and [31], (1.3) has a unique radial solution).

The method of our construction evolves from that of [11], [28] and [29] on the semi-classical (i.e. for small parameter $h$ ) solution of the nonlinear Schrödinger equation

$$
\frac{h^{2}}{2} \Delta U-(V-E) U+U^{p}=0
$$

in $R^{N}$ where $V$ is a potential function and $E$ is a real constant. The method of Lyapunov-Schmidt reduction was used in [11], [28] and [29] to construct solutions of (1.4) close to nondegenerate critical points of $V$ for $h$ sufficiently small.

Following the strategy of [11], [28] and [29] we shall construct a solution $v_{\epsilon}$ of (1.2) with maxima near $M$ given nondegenerate critical points of the mean curvature $P_{0, i}$ on $\partial \Omega$ by taking the sum of $M$ functions each having a peak lying on the boundary and being close to $P_{0, i}$ for $i=1,2, \ldots, M$. 
Heuristically, for each of these functions we rescale (1.2) to obtain

$$
\left\{\begin{array}{l}
\Delta u_{\epsilon}-m u_{\epsilon}+h\left(u_{\epsilon}\right)-\frac{1}{\left|\Omega_{\epsilon}\right|} \int_{\Omega_{\epsilon}} h\left(u_{\epsilon}\right)=0 \quad \text { in } \Omega_{\epsilon}, \\
\frac{\partial u_{\epsilon}}{\partial \nu_{\epsilon}}=0 \quad \text { on } \partial \Omega_{\epsilon}
\end{array}\right.
$$

where $u_{\epsilon}=v_{\epsilon}(\epsilon y), \Omega_{\epsilon}=\epsilon^{-1} \Omega$ (assuming $P_{0, i}=0$, the origin) and $\nu_{\epsilon}$ is the unit outer normal to $\partial \Omega_{\epsilon}$.

An immediate though formal calculation shows that $u_{\epsilon} \rightarrow V$ as $\epsilon \rightarrow 0$ where $V$ is the unique solution of

$$
\left\{\begin{array}{l}
\Delta w-m w+h(w)=0 \quad \text { in } R_{+}^{N}, \\
w>0 \quad \text { in } R_{+}^{N}, \\
\frac{\partial w}{\partial y_{N}}=0 \quad \text { on } R^{N-1} \times\{0\}
\end{array}\right.
$$

with $V(0)=\max _{R_{+}^{N}} V$. Therefore the ground state solution $V$ restricted to $R_{+}^{N}$ can be an approximate solution for $u_{\epsilon}$. Since the linearized problem arising from (1.6) has the $(N-1)$-dimensional kernel $\operatorname{span}\left\{\frac{\partial V}{\partial y_{1}}, \ldots, \frac{\partial V}{\partial y_{N-1}}\right\}$ we first "solve" (1.6) up to this kernel and then use the nondegeneracy of $H\left(P_{i}\right)$ to take care of the kernel separately.

The proof of Theorem 1.1 also works for the the following singular perturbation problem

$$
\begin{cases}\epsilon^{2} \Delta v+g(v)=0 & \text { in } \Omega \\ \frac{\partial v}{\partial \nu}=0 & \text { on } \partial \Omega .\end{cases}
$$

Furthermore, the proof of Theorem 1.1 can be adapted to deal with nonlinearities satisfying the following conditions:

(g1) $g(0)=0, g^{\prime}(0)=-m<0$

(g2) $g \in C^{1+\sigma}\left(R^{+}\right), g(u)=-m u+h(u)$ where $h$ satisfies

$$
h(u)=O\left(|u|^{p_{1}}\right), h^{\prime}(u)=O\left(|u|^{p_{2}-1}\right) \text { as }|u| \rightarrow \infty
$$

for some $1<p_{1}, p_{2}$ and there exists $1<p_{3}$ such that

$$
\left|h_{u}(u+\phi)-h_{u}(u)\right| \leq\left\{\begin{array}{l}
C|\phi|^{p_{3}-1} \text { if } p_{3}>2 \\
C\left(|\phi|+|\phi|^{p_{3}-1}\right) \text { if } p_{3} \leq 2
\end{array}\right.
$$

(g3) The equation

$$
\left\{\begin{array}{l}
\triangle w+g(w)=0 \quad \text { in } \mathbb{R}^{\mathbb{N}} \\
w>0, w(0)=\max _{z \in R^{n}} w(z) \\
w \rightarrow 0 \text { at } \infty
\end{array}\right.
$$


has a unique solution $V(y)$ (by the results of [13], $V$ is radial, i.e., $V=V(r)$ and $V^{\prime}<0$ for $\left.r=|y| \neq 0\right)$ and $V$ is nondegenerate. Namely the operator

$$
L:=\triangle+g^{\prime}(V)
$$

is invertible in the space $H_{r}^{2}\left(R^{N}\right):=\left\{u=u(|y|) \in H^{2}\left(R^{N}\right)\right\}$.

It is easy to see that for the Cahn-Hillard equation conditions (g1), (g2) and (g3) are satisfied. Two other important examples are the following.

Example 1 (chemotaxis and pattern formation): $g(u)=-u+u^{p}$ where $1<p<\left(\frac{N+2}{N-2}\right)_{+}\left(=\infty\right.$ if $N=2 ;=\frac{N+2}{N-2}$ if $\left.N>2\right)$. It is easy to see that $g$ satisfies (g1), (g2) and (g3). Hence multiple boundary spike solutions can be constructed for multiple nondegenerate critical points of the mean curvature. This problem arises from the Keller-Segal model in chemotaxis and the Gierer-Meinhardt system in pattern formation (see [25], [26] and the references therein). Single boundary spike solutions have been extensively studied by [25], [26], [33], etc. In [12], Gui used variational methods to construct multiple boundary spike solutions at strict local maximum points of the mean curvature. Our result in this paper is the first result in constructing multiple boundary spike solutions in the general situation.

Example 2 (population dynamics and chemical reaction theory): $g(u)=u(u-a)(1-u)$ where $0<a<\frac{1}{2}$. This is a famous model from population dynamics and chemical reaction theory (see [4], [19], [32]). By the result of [14], $g$ satisfies (g1)-(g3). Hence single and multiple boundary spike solutions can be constructed. This is the first result in constructing boundary spike solutions for this nonlinearity. Note that the methods of [12] and [25] cannot be applied here since $g$ does not satisfy the conditions in those papers.

Other nonlinearities satisfying $(g 1),(g 2)$ and $(g 3)$ can be found in [8].

The existence of spike layer solutions as well as the location and the profile of the peaks for other problems arising in various models such as chemotaxis, pattern formation, chemical reactor theory, etc. have been studied by Lin, $\mathrm{Ni}$, Pan, and Takagi $[21,24,25,26]$ for the Neumann problem and by $\mathrm{Ni}$ and 
Wei [27] for the Dirichlet problem. However, they consider only least-energy, hence single-peak solutions.

In this paper, we prove Theorem 1.1 for the Cahn-Hilliard case. The arguments can be easily modified to deal with the other cases of more general nonlinearities.

The paper is organized as follows. Notation, preliminaries and some useful estimates are explained in Section 2. Section 3 contains the setup of our problem and we solve (1.2) up to approximate kernel and cokernel, respectively. Finally in Section 4 we solve the reduced problem.

Acknowledgement. The first author would like to thank Professor WeiMing Ni for his enlightening discussions. The second author would like to acknowledge discussions with Professor Amy Novick-Cohen. Part of the work is inspired by some related work of Professor Wei-Ming $\mathrm{Ni}$ and Professor Yong-Geun Oh. This research was done while the second author visited the Department of Mathematics, The Chinese University of Hong Kong. It is supported by a Direct Grant from The Chinese University of Hong Kong and by a grant of the European Union (contract ERBCHBICT930744).

\section{Technical Analysis}

In this section we introduce a projection and derive some useful estimates. Throughout the paper we shall use the letter $C$ to denote a generic positive constant which may vary from term to term. We denote $R_{+}^{N}=$ $\left\{\left(x^{\prime}, x_{N}\right) \mid x_{N}>0\right\}$. Let $V$ be the unique solution of (1.3).

Let $P \in \partial \Omega$. We can define a diffeomorphism straightening the boundary in a neighborhood of $P$. After rotation of the coordinate system we may assume that the inward normal to $\partial \Omega$ at $P$ is pointing in the direction of the

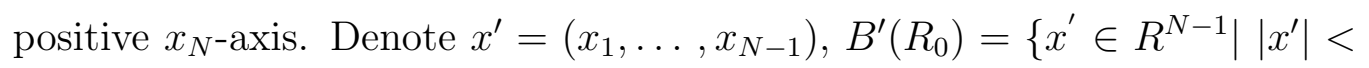
$\left.R_{0}\right\}, B\left(P, R_{0}\right)=\left\{x \in R^{N}|| x-P \mid<R_{0}\right\}$, and $\Omega_{1}=\Omega \cap B\left(P, R_{0}\right)=$ $\left\{\left(x^{\prime}, x_{N}\right) \in B\left(P, R_{0}\right) \mid x_{N}-P_{N}>\rho\left(x^{\prime}-P^{\prime}\right)\right\}$. Then, since $\partial \Omega$ is smooth, we

can find a constant $R_{0}>0$ such that $\partial \Omega \cap \overline{\Omega_{1}}$ can be represented by the graph of a smooth function $\rho_{P}: B^{\prime}\left(R_{0}\right) \rightarrow R$ where $\rho_{P}(0)=0, \nabla \rho_{P}(0)=0$. 
From now on we omit the use of $P$ in $\rho_{P}$ and write $\rho$ instead if this can be done without causing confusion. The sum of the principal curvatures of $\partial \Omega$ at $P$ is $H(P)=\sum_{i=1}^{N-1} \rho_{i i}(0)$ where

$$
\rho_{i}=\frac{\partial \rho}{\partial x_{i}}, \quad i=1, \ldots, N-1
$$

and higher derivatives will be defined in the same way. By Taylor expansion we have

$$
\begin{gathered}
\rho\left(x^{\prime}-P^{\prime}\right)=\frac{1}{2} \sum_{i, j=1}^{N-1} \rho_{i j}(0)\left(x_{i}-P_{i}\right)\left(x_{j}-P_{j}\right) \\
+\frac{1}{6} \sum_{i, j, k=1}^{N-1} \rho_{i j k}(0)\left(x_{i}-P_{i}\right)\left(x_{j}-P_{j}\right)\left(x_{k}-P_{k}\right)+\mathcal{O}\left(\left|x^{\prime}-P^{\prime}\right|^{4}\right)
\end{gathered}
$$

In the following we use $\rho_{\alpha}$ to denote the multiple differentiation $\frac{\partial^{|\alpha|} \rho}{\partial x^{\alpha}}$ where $\alpha$ is a multiple index.

For a smooth bounded domain $U$ we now introduce a projection $P_{U}$ of $H^{2}(U)$ onto $\left\{v \in H^{2}(U) \mid \partial v / \partial \nu=0\right.$ at $\left.\partial U\right\}$ as follows: For $v \in H^{2}(U)$ let $w=P_{U} v$ be the unique solution of the boundary value problem

$$
\begin{cases}\Delta w-m w+h(v)=0 & \text { in } U \\ \frac{\partial w}{\partial \nu}=0 & \text { on } \partial U\end{cases}
$$

Let $h_{\epsilon, P}(x)=V\left(\frac{x-P}{\epsilon}\right)-P_{\Omega_{\epsilon, P}}\left[V\left(\frac{x-P}{\varepsilon}\right)\right]$ where

$$
\Omega_{\epsilon, P}=\left\{z \in R^{n} \mid \epsilon z+P \in \Omega\right\} .
$$

Then $h_{\epsilon, P}$ satisfies

$$
\left\{\begin{array}{l}
\epsilon^{2} \Delta v-m v=0 \quad \text { in } \Omega \\
\frac{\partial v}{\partial \nu}=\frac{\partial V}{\partial \nu} \quad \text { on } \partial \Omega
\end{array}\right.
$$

We denote

$$
\|v\|_{\epsilon}^{2}=\epsilon^{-N} \int_{\Omega}\left[\epsilon^{2}|\nabla v|^{2}+m v^{2}\right]
$$

For $x \in \Omega_{1}$ set now

$$
\left\{\begin{array}{l}
\epsilon y^{\prime}=x^{\prime}-P^{\prime} \\
\epsilon y_{N}=x_{N}-P_{N}-\rho\left(x^{\prime}-P^{\prime}\right) .
\end{array}\right.
$$

Furthermore, for $x \in \Omega_{1}$ we introduce the transformation $T$ by

$$
\left\{\begin{array}{l}
T_{i}\left(x^{\prime}\right)=x_{i}, \\
T_{N}\left(x^{\prime}\right)=x_{N}-P_{N}-\rho\left(x^{\prime}-P^{\prime}\right) .
\end{array}\right.
$$


Note that then

$$
y=\frac{1}{\epsilon} T(x) .
$$

Let $v_{1}$ be the unique solution of

$$
\left\{\begin{array}{l}
\Delta v-m v=0 \quad \text { in } R_{+}^{N}, \\
\frac{\partial v}{\partial y_{N}}=-\frac{V^{\prime}}{|y|} \frac{1}{2} \sum_{i, j=1}^{N-1} \rho_{i j}(0) y_{i} y_{j} \quad \text { on } \partial R_{+}^{N}
\end{array}\right.
$$

where $V^{\prime}$ is the radial derivative of $V$, meaning that $V^{\prime}=V_{r}(r)$, and $r=$ $\left|\frac{x-P}{\epsilon}\right|$. Let $v_{2}$ be the unique solution of

$$
\begin{cases}\Delta v-m v-2 \sum_{i, j=1}^{N-1} \rho_{i j}(0) y_{i} \frac{\partial^{2} v_{1}}{\partial y_{j} \partial y_{N}}=0 & \text { in } R_{+}^{N}, \\ \frac{\partial v}{\partial y_{N}}=\sum_{i, j=1}^{N-1} \rho_{i j}(0) y_{i} \frac{\partial v_{1}}{\partial y_{j}} \quad \text { on } \partial R_{+}^{N} . & \end{cases}
$$

Let $v_{3}$ be the unique solution of

$$
\left\{\begin{array}{l}
\Delta v-m v=0 \quad \text { in } R_{+}^{N}, \\
\frac{\partial v}{\partial y_{N}}=-\frac{V^{\prime}}{|y|} \frac{1}{3} \sum_{i, j, k=1}^{N-1} \rho_{i j k}(0) y_{i} y_{j} y_{k} \quad \text { on } \partial R_{+}^{N} .
\end{array}\right.
$$

Note that $v_{1}, v_{2}$ are even functions in $y^{\prime}=\left(y_{1}, \ldots, y_{N-1}\right)$ and $v_{3}$ is an odd function in $y^{\prime}=\left(y_{1}, \ldots, y_{N-1}\right)$ (i.e. $v_{1}\left(y^{\prime}, y_{N}\right)=v_{1}\left(-y^{\prime}, y_{N}\right), v_{3}\left(y^{\prime}, y_{N}\right)=$ $\left.-v_{3}\left(-y^{\prime}, y_{N}\right)\right)$. Moreover, it is easy to see that $\left|v_{1}\right|,\left|v_{2}\right|,\left|v_{3}\right| \leq C e^{-\mu|y|}$ for some $0<\mu<\sqrt{m}$. Let $\chi(x)$ be a smooth cutoff function such that $\chi(x)=$ 1 , for $x \in B\left(0, R_{0}-\delta\right)$ and $\chi(x)=0$ for $x \in B\left(0, R_{0}\right)^{C}$ (where $\delta$ is a positive number). Set

$$
h_{\epsilon, P}(x)=\epsilon v_{1}(y) \chi(x-P)+\epsilon^{2}\left(v_{2}(y) \chi(x-P)+v_{3}(y) \chi(x-P)\right)+\epsilon^{3} \Psi_{\epsilon, P}(x) .
$$

Then we have the following proposition.

Proposition 2.1. The remainder $\Psi_{\epsilon, P}$ satisfies

$$
\left\|\Psi_{\epsilon, P}\right\|_{\epsilon} \leq C
$$

Proof. Proposition 2.1 was proved in [34] by Taylor expansion and a rigorous estimate of the remainder using estimates for elliptic partial differential equations.

Similarly, we know from [34] that the following proposition is true. 
Proposition 2.2. We have

$$
\left[\frac{\partial V}{\partial \tau_{P_{j}}}-\frac{\partial P_{\Omega_{\epsilon, P}} V}{\partial \tau_{P_{j}}}\right]\left(\frac{x-P}{\epsilon}\right)=w_{1}(y) \chi(x-P)+\epsilon w_{2}^{\epsilon}(x)
$$

where $w_{1}$ satisfies

$$
\left\{\begin{array}{l}
\Delta v-m v=0 \quad \text { in } R_{+}^{N}, \\
\frac{\partial v}{\partial y_{N}}=-\frac{1}{2}\left(\frac{V^{\prime \prime}}{|y|^{2}}-\frac{V^{\prime}}{|y|^{3}}\right) \sum_{k, l=1}^{N-1} \rho_{k l}(0) y_{k} y_{l} y_{j}-\frac{V^{\prime}}{|y|} \sum_{k=1}^{N-1} \rho_{j k}(0) y_{k} \text { on } \partial R_{+}^{N}
\end{array}\right.
$$

and

$$
\left\|w_{2}^{\epsilon}\right\|_{\epsilon} \leq C
$$

Note that $\left|w_{1}\right| \leq C \exp (-\mu|y|)$ for some $\mu<\sqrt{m}$ where $w_{1}$ is an odd function in $y^{\prime}$ and that $\left|w_{2}\right| \leq C \exp (-\mu|y|)$ for some $\mu<\sqrt{m}$.

Define the linear operator $L_{0}$ by

$$
L_{0} u=\Delta u-m u+h^{\prime}(V) u
$$

for

$$
u \in H_{N}^{2}\left(R_{+}^{N}\right)=\left\{u \in H^{2}\left(R_{+}^{N}\right), \frac{\partial u}{\partial y_{N}}=0 \text { on } \partial R_{+}^{N}\right\} .
$$

We have the following statement.

Lemma 2.3. The kernel of $L_{0}$ satisfies

$$
\operatorname{Ker}\left(L_{0}\right) \cap H_{N}^{2}\left(R_{+}^{N}\right)=\operatorname{span}\left\{\frac{\partial V}{\partial y_{1}}, \ldots, \frac{\partial V}{\partial y_{N-1}}\right\}
$$

Proof. See Lemma 4.2 in [26]. 


\section{Reduction to Finite Dimensions}

In this section we use the Lyapunov-Schmidt method to reduce the problem to finite dimensions.

Let $P \in \bar{\Omega}$,

$$
\Omega_{\epsilon}=\left\{z \in R^{N} \mid \epsilon z \in \Omega\right\}
$$

and

$$
\Omega_{\epsilon, P}=\left\{z \in R^{N} \mid \epsilon z+P \in \Omega\right\} .
$$

Let $H_{N}^{2}\left(\Omega_{\epsilon}\right)$ be the Hilbert space defined by

$$
H_{N}^{2}\left(\Omega_{\epsilon}\right)=\left\{u \in H^{2}\left(\Omega_{\epsilon}\right) \mid \frac{\partial u}{\partial \nu_{\epsilon}}=0 \text { on } \partial \Omega_{\epsilon}\right\} .
$$

Define

$$
\tilde{S}_{\epsilon}(u)=\Delta u-m u+h(u)-\frac{1}{\left|\Omega_{\epsilon}\right|} \int_{\Omega_{\epsilon}} h(u)
$$

for $u \in H_{N}^{2}\left(\Omega_{\epsilon}\right)$. Then equation (1.2) is equivalent to

$$
\tilde{S}_{\epsilon}(u)=0, u \in H_{N}^{2}\left(\Omega_{\epsilon}\right) .
$$

Fix $\mathbf{P}=\left(P_{1}, \ldots, P_{M}\right)$ with $P_{i} \in \partial \Omega$. For the rest of this section we fix a small $\delta>0$ such that

$$
\min _{i, j=1, \ldots, M, i \neq j} \operatorname{dist}\left(P_{i}, P_{j}\right) \geq \delta
$$

We set

$$
\begin{gathered}
P V_{i}(y)=P_{\Omega_{\varepsilon, P_{i}}} V\left(y-\frac{P_{i}}{\epsilon}\right), \\
V_{i}(y)=V\left(y-\frac{P_{i}}{\varepsilon}\right)
\end{gathered}
$$

and

$$
u=\sum_{i=1}^{M} P V_{i}+\Phi_{\varepsilon, \mathbf{P}} .
$$

To solve (1.2) we first consider the linear operator

$$
\begin{aligned}
& \tilde{L}_{\epsilon}: u \mapsto \Delta u-m u+h^{\prime}\left(\sum_{i=1}^{M} P V_{i}\right) u, \\
& H_{N}^{2}\left(\Omega_{\epsilon}\right) \rightarrow L^{2}\left(\Omega_{\epsilon}\right)
\end{aligned}
$$


Using integration by parts it is easy to see that the cokernel of $\tilde{L}_{\epsilon}$ coincides with its kernel. We choose the approximate cokernel $\mathcal{C}_{\epsilon, \mathbf{P}}$ and kernel $\mathcal{K}_{\epsilon, \mathbf{P}}$ as

$$
\begin{gathered}
\mathcal{C}_{\epsilon, \mathbf{P}}=\mathcal{K}_{\epsilon, \mathbf{P}} \\
=\operatorname{span}\left\{\frac{\partial P V_{i}}{\partial \tau_{P_{i, j}}} \mid i=1, \ldots, M, j=1, \ldots, N-1\right\} .
\end{gathered}
$$

Let $\pi_{\epsilon, \mathbf{P}}$ denote the projection of $L^{2}\left(\Omega_{\epsilon}\right)$ onto $\mathcal{C}_{\epsilon, \mathbf{P}}^{\perp}$. Our goal in this section is to show that the equation

$$
\pi_{\epsilon, \mathbf{P}} \circ \tilde{S}_{\epsilon}\left(\sum_{i=1}^{M} P V_{i}+\Phi_{\epsilon, \mathbf{P}}\right)=0
$$

has a unique solution $\Phi_{\epsilon, \mathbf{P}} \in \mathcal{K}_{\epsilon, \mathbf{P}}^{\perp}$ if $\epsilon$ is small enough.

As a preparation in the following two propositions we show invertibility of the corresponding linearized operator.

Proposition 3.1. Let $L_{\epsilon, \mathbf{P}}=\pi_{\epsilon, \mathbf{P}} \circ \tilde{L}_{\epsilon}$. There exist positive constants $\bar{\epsilon}, \lambda$ such that for all $\epsilon \in(0, \bar{\epsilon})$ and all $\mathbf{P}=\left(P_{1}, \ldots, P_{M}\right)$ with $P_{1}, \ldots, P_{M} \in \partial \Omega$ and $\min _{i, j=1, \ldots, M, i \neq j} \operatorname{dist}\left(P_{i}, P_{j}\right) \geq \delta$ :

$$
\left\|L_{\epsilon, \mathbf{P}} \Phi\right\|_{L^{2}\left(\Omega_{\epsilon}\right)} \geq \lambda\|\Phi\|_{H^{2}\left(\Omega_{\epsilon}\right)}
$$

for all $\Phi \in \mathcal{K}_{\epsilon, \mathbf{P}}^{\perp}$

Proposition 3.2. There exists a positive constant $\tilde{\epsilon}$ such that for all $\epsilon \in$ $(0, \tilde{\epsilon})$ and all $\mathbf{P}=\left(P_{1}, \ldots, P_{M}\right)$ with $P_{1}, \ldots, P_{M} \in \partial \Omega$ and

$$
\min _{i, j=1, \ldots, M, i \neq j} \operatorname{dist}\left(P_{i}, P_{j}\right) \geq \delta
$$

the map

$$
L_{\epsilon, \mathbf{P}}=\pi_{\epsilon, \mathbf{P}} \circ \tilde{L}_{\epsilon}: \mathcal{K}_{\epsilon, \mathbf{P}}^{\perp} \rightarrow \mathcal{C}_{\epsilon, \mathbf{P}}^{\perp}
$$

is surjective. 
Proof of Proposition 3.1: We will follow the method used in [11], [28], [29] and [34]. Suppose that (3.1) is false. Then there exist sequences $\left\{\epsilon_{k}\right\},\left\{\mathbf{P}_{\mathbf{k}}\right\}=\left\{\left(P_{1, k}, \ldots, P_{M, k}\right)\right\}$ and $\left\{\Phi_{k}\right\}$ for $k=1,2, \ldots$ with the following properties: $\epsilon_{k}>0$ and $P_{i, k} \in \partial \Omega$ with

$$
\min _{i, j=1, \ldots, M, i \neq j} \operatorname{dist}\left(P_{i, k}, P_{j, k}\right)>\delta
$$

such that $\Phi_{k} \in \mathcal{K}_{\epsilon_{k}, \mathbf{P}_{k}}^{\perp}$ and

$$
\begin{aligned}
& \epsilon_{k} \rightarrow 0 \\
& \mathbf{P}_{\mathbf{k}} \rightarrow \mathbf{P} \\
& \left\|L_{\epsilon^{6} k}, \mathbf{P}_{\mathbf{k}} \Phi_{k}\right\|_{L^{2}} \rightarrow 0 \\
& \left\|\Phi_{k}\right\|_{H^{2}}=1 \quad \text { for } k=1,2, \ldots
\end{aligned}
$$

For $j=1,2, \ldots, N-1$ denote

$$
e_{i j, k}=\frac{\partial}{\partial \tau_{\left(P_{i, k}\right)_{j}}} P V_{i, k} /\left\|\frac{\partial}{\partial \tau_{\left(P_{i, k}\right)_{j}}} P V_{i, k}\right\|_{L^{2}\left(\Omega_{\epsilon_{k}}\right)} .
$$

Note that

$$
<e_{i_{1} j_{1}, k}, e_{i_{2} j_{2}, k}>=\delta_{i_{1} i_{2}} \delta_{j_{1} j_{2}}+\mathcal{O}\left(\epsilon_{k}\right) \quad \text { as } k \rightarrow \infty
$$

by Proposition 2.3 and because of the symmetry of the function $w_{1}$ which was defined in (2.9). Here $\delta_{i_{1} i_{2}}$ is the Kronecker symbol. Furthermore because of (3.4) we deduce that

$$
\left\|\tilde{L}_{\epsilon_{k}} \Phi_{k}\right\|_{L^{2}}^{2}-\sum_{i=1}^{M} \sum_{j=1}^{N-1}\left(\int_{\Omega_{\epsilon_{k}}} \tilde{L}_{\epsilon_{k}} \Phi_{k} e_{i j, k}\right)^{2} \rightarrow 0
$$

as $k \rightarrow \infty$. Let $\Omega_{0}, \chi, \rho$ and $T$ be the same as in Section 2. Then $T$ has an inverse $T^{-1}$ such that

$$
T^{-1}: T\left(B\left(P, R_{0}\right) \cap \bar{\Omega}\right) \rightarrow B\left(P, R_{0}\right) \cap \bar{\Omega} .
$$

Recall that $\epsilon y=T(x)$. We use the notation $T^{(i)}$ if $P$ is replaced by $P_{i}$. We introduce new sequences $\left\{\varphi_{i, k}\right\}$ by

$$
\varphi_{i, k}(y)=\chi\left(\frac{1}{\epsilon^{k}}\left(T^{(i)}\right)^{-1}\left(\epsilon_{k} y\right)\right) \Phi_{k}\left(\left(T^{(i)}\right)^{-1}\left(\epsilon_{k} y\right)\right)
$$


for $y \in R_{+}^{N}$. Since $T^{(i)}$ and $\left(T^{(i)}\right)^{-1}$ have bounded derivatives it follows from (3.5) and the smoothness of $\chi$ that

$$
\left\|\varphi_{i, k}\right\|_{H^{2}\left(R_{+}^{N}\right)} \leq C
$$

for all $k$ sufficiently large. On the other hand,

$$
\left\|\varphi_{i, k}\right\|_{H^{2}\left(R_{+}^{N} \backslash B(0, R)\right)} \rightarrow 0 \quad \text { as } R \rightarrow \infty
$$

uniformly in $k$ for all $k$ large enough. Therefore there exists a subsequence, again denoted by $\left\{\varphi_{i, k}\right\}$ which converges weakly in $H^{2}\left(R_{+}^{N}\right)$ to a limit $\varphi_{i, \infty}$ as $k \rightarrow \infty$. We are now going to show that $\varphi_{i, \infty} \equiv 0$. As a first step we deduce that

$$
\int_{R_{+}^{N}} \varphi_{i, \infty} \frac{\partial V}{\partial y_{j}}=0 \quad \text { for } j=1, \ldots, N-1 .
$$

Noting that $\operatorname{det} D T=\operatorname{det} D T^{-1}=1$ this statement is shown as follows

$$
\begin{gathered}
\int_{R_{+}^{N}} \varphi_{i, k}(y)\left[\frac{\partial P V_{i, k}}{\partial \tau_{\left(P_{i, k}\right)_{j}}}\left(\frac{\left(T^{(i)}\right)^{-1}\left(\epsilon_{k} y\right)}{\epsilon_{k}}\right)\right] d y \\
=\epsilon_{k}^{-N} \int_{\Omega_{0}} \chi\left(x-P_{i, k}\right) \Phi_{k}\left(\frac{x}{\epsilon_{k}}\right) \frac{\partial P V_{i, k}}{\partial \tau_{\left(P_{i, k}\right)_{j}}\left(\frac{x-P_{i, k}}{\epsilon_{k}}\right) d x} \\
=\epsilon_{k}^{-N} \int_{\Omega} \Phi_{k}\left(\frac{x}{\epsilon_{k}}\right) \frac{\partial P V_{i, k}}{\partial \tau_{\left(P_{i, k}\right)_{j}}}\left(\frac{x-P_{i, k}}{\epsilon_{k}}\right) \\
-\epsilon_{k}^{-N} \int_{\Omega \backslash \Omega_{0}} \Phi_{k}\left(\frac{x}{\epsilon_{k}}\right) \frac{\partial P V_{i, k}}{\partial \tau_{\left(P_{i, k}\right)_{j}}\left(\frac{x-P_{i, k}}{\epsilon_{k}}\right)} \\
-\epsilon_{k}^{-N} \int_{\Omega_{0}}\left[1-\chi\left(x-P_{i, k}\right)\right] \Phi_{k}\left(\frac{x}{\epsilon_{k}}\right) \frac{\partial P V_{i, k}}{\partial \tau_{\left(P_{i, k}\right)_{j}}}\left(\frac{x-P_{i, k}}{\epsilon_{k}}\right) \\
=0-\epsilon_{k}^{-N} \int_{\Omega \backslash \Omega_{0}} \Phi_{k}\left(\frac{x}{\epsilon_{k}}\right)\left[\frac{\partial V}{\partial\left(P_{i, k}\right)_{j}}\left(\frac{x-P_{i, k}}{\epsilon_{k}}\right)-\frac{\partial P V_{i, k}}{\partial \tau_{\left(P_{i, k}\right)_{j}}}\left(\frac{x-P_{i, k}}{\epsilon_{k}}\right)\right] \\
-\epsilon_{k}^{-N}\left[1-\chi\left(x-P_{i, k}\right)\right] \Phi_{k}\left(\frac{x}{\epsilon_{k}}\right)\left[\frac{\partial V}{\partial\left(P_{i, k}\right)_{j}}\left(\frac{x-P_{i, k}}{\epsilon_{k}}\right)-\frac{\partial P V_{i, k}}{\partial \tau_{\left(P_{i, k}\right)_{j}}}\left(\frac{x-P_{i, k}}{\epsilon_{k}}\right)\right] \\
-\epsilon_{k}^{-N} \int_{\Omega \backslash \Omega_{0}} \Phi_{k}\left(\frac{x}{\epsilon_{k}}\right) \frac{\partial V}{\partial\left(P_{i, k}\right)_{j}}\left(\frac{x-P_{i, k}}{\epsilon_{k}}\right) \\
-\epsilon_{k}^{-N} \int_{\Omega_{0}}\left[1-\chi\left(x-P_{i, k}\right)\right] \Phi_{k}\left(\frac{x}{\epsilon_{k}}\right) \frac{\partial V}{\partial\left(P_{i, k}\right)_{j}}\left(\frac{x-P_{i, k}}{\epsilon_{k}}\right)
\end{gathered}
$$

where $\Omega_{0}$ is as defined in section 2. In the last expression the first two terms tend to zero as $k \rightarrow \infty$ since $\epsilon_{k}{ }^{-N} \Phi_{k}$ is bounded in $L^{2}(\Omega)$ and $[\ldots] \rightarrow 0$ 
strongly in $L^{2}(\Omega)$. The last two terms tend to zero as $k \rightarrow \infty$ because of the exponential decay of $\partial V / \partial\left(P_{i, k}\right)_{j}$ at infinity.

We conclude that

$$
\begin{gathered}
\limsup _{k \rightarrow \infty}\left|\int_{R_{+}^{N}} \varphi_{i, k}\left(y-P_{i, k} / \varepsilon_{k}\right)\left[\frac{\partial P V_{i, k}}{\partial \tau_{\left(P_{i, k}\right)}}\left(\frac{\left(T^{(i)}\right)^{-1}\left(\epsilon_{k} y\right)}{\epsilon_{k}}\right)\right]\right|=0 \\
\text { for } i=1, \ldots, M \text { and } j=1, \ldots, N-1 .
\end{gathered}
$$

This implies (3.8).

Let $\mathcal{K}_{0}$ and $\mathcal{C}_{0}$ be the kernel and cokernel, respectively, of the linear operator $S_{0}^{\prime}(V)$ which is the Fréchet derivative at $V$ of

$$
\begin{gathered}
S_{0}(v)=\Delta v-m v+h(v), \\
S_{0}: H_{N}^{2}\left(R_{+}^{N}\right) \rightarrow L^{2}\left(R_{+}^{N}\right)
\end{gathered}
$$

where

Note that

$$
H_{N}^{2}\left(R_{+}^{N}\right)=\left\{u \in H_{N}^{2}\left(R_{+}^{N}\right) \mid \frac{\partial u}{\partial y_{N}}=0\right\} .
$$

$$
\mathcal{K}_{0}=\mathcal{C}_{0}=\operatorname{span}\left\{\frac{\partial V}{\partial y_{j}} \mid j=1, \ldots, N-1\right\} .
$$

Equation (3.8) implies that $\varphi_{i, \infty} \in \mathcal{K}_{0}^{\perp}$. By the exponential decay of $V$ and by (3.4) we have after possibly taking a further subsequence that

$$
\Delta \varphi_{i, \infty}-m \varphi_{i, \infty}+h^{\prime}(V) \varphi_{i, \infty}=0,
$$

that is to say $\varphi_{i, \infty} \in \mathcal{K}_{0}$. Therefore $\varphi_{i, \infty}=0$ for $i=1, \ldots, M$.

Hence

$$
\varphi_{i, k} \rightarrow 0 \quad \text { weakly in } H^{2}\left(R_{+}^{N}\right)
$$

as $k \rightarrow \infty$. By the definition of $\varphi_{i, k}$ we get $\Phi_{k} \rightarrow 0$ in $H^{2}$ and

$$
\left\|h^{\prime}\left(\sum_{i=1}^{M} P V_{i, k}\right) \Phi_{k}\right\|_{L^{2}} \rightarrow 0 \quad \text { as } k \rightarrow \infty .
$$

Furthermore,

$$
\left\|(\Delta-m) \Phi_{k}\right\|_{L^{2}} \rightarrow 0 \quad \text { as } k \rightarrow \infty
$$

Since

$$
\int_{\Omega_{\epsilon_{k}}}\left|\nabla \Phi_{k}\right|^{2}+m \Phi_{k}^{2}=\int_{\Omega_{\epsilon_{k}}}\left[(m-\Delta) \Phi_{k}\right] \Phi_{k}
$$




$$
\leq C\left\|(\Delta-m) \Phi_{k}\right\|_{L^{2}}
$$

we have

$$
\left\|\Phi_{k}\right\|_{H^{1}} \rightarrow 0 \quad \text { as } k \rightarrow \infty .
$$

In summary we conclude that

$$
\left\|\Delta \Phi_{k}\right\|_{L^{2}} \rightarrow 0 \text { and }\left\|\Phi_{k}\right\|_{H^{1}} \rightarrow 0
$$

Using (3.11) and the elliptic regularity estimate

$$
\left\|\Phi_{k}\right\|_{H^{2}} \leq C\left(\left\|\Delta \Phi_{k}\right\|_{L^{2}}+\left\|\Phi_{k}\right\|_{H^{1}}\right)
$$

for $\Phi_{k} \in H_{N}^{2}$ (a proof can be found in Appendix B of [34]) we deduce that

$$
\left\|\Phi_{k}\right\|_{H^{2}} \rightarrow 0 \quad \text { as } k \rightarrow \infty \text {. }
$$

This contradicts the assumption

$$
\left\|\Phi_{k}\right\|_{H^{2}}=1
$$

and the proof of Proposition 3.1 is completed.

Proof of Proposition 3.2: Assume that the statement is not true. Then there exist sequences $\left\{\epsilon_{k}\right\},\left\{\mathbf{P}_{\mathbf{k}}\right\}=\left\{\left(P_{1, k}, \ldots, P_{M, k}\right)\right\}$ with $\varepsilon_{k}>0$ and $P_{i, k} \in \partial \Omega$ and

$$
\min _{i, j=1, \ldots, M, i \neq j} \operatorname{dist}\left(P_{i, k}, P_{j, k}\right) \geq \delta
$$

such that $L_{\epsilon_{k}, \mathbf{P}_{\mathbf{k}}}: \mathcal{K}_{\epsilon_{k}, \mathbf{P}_{\mathbf{k}}}^{\perp} \rightarrow \mathcal{C}_{\epsilon_{k}, P_{k}}^{\perp}$ is not surjective. Furthermore, $\epsilon_{k} \rightarrow 0$ as $k \rightarrow \infty$ and $\mathbf{P}_{\mathbf{k}} \rightarrow \mathbf{P}$. for all $k$. Let $K_{\epsilon, \mathbf{P}}$ and $C_{\epsilon, \mathbf{P}}$ be the (exact) kernel and cokernel of $\tilde{L}_{\epsilon}$, respectively. Then for $k=1,2, \ldots$ there exists $\Phi_{k} \in C_{\epsilon_{k}, P_{k}}$ such that

$$
\left\|\Phi_{k}\right\|_{L^{2}\left(\Omega_{\epsilon_{k}}\right)}=1
$$

and

$$
\int_{\Omega_{\epsilon_{k}}} \Phi_{k} \frac{\partial P V_{i, k}}{\partial \tau\left(P_{i, k}\right)_{j}}=0 \text { for } i=1, \ldots, M, j=1, \ldots, N-1 .
$$

Since $\Phi_{k} \in C_{\epsilon_{k}, P_{k}}$ we have

$$
\int_{\Omega_{\epsilon_{k}}}\left(\Delta \varphi-\varphi+f^{\prime}\left(\sum_{i=1}^{M} P V_{i, k}\right) \varphi\right) \Phi_{k}=0
$$


for all $\varphi \in H_{N}^{2}\left(\Omega_{\epsilon_{k}}\right)$. Now integration by parts gives

$$
\Delta \Phi_{k}-m \Phi_{k}+h^{\prime}\left(\sum_{i=1}^{M} P V_{i, k}\right) \Phi_{k}=0
$$

and because of the elliptic estimate (3.12) it follows that

$$
\left\|\Phi_{k}\right\|_{H^{2}} \leq C
$$

for some constant $C$ independent of $k$. Extract a subsequence (again denoted by $\left.\left\{\Phi_{i, k}\right\}\right)$ such that $\varphi_{i, k}$ as defined in (3.7) converges weakly in $H^{2}\left(R_{+}^{N}\right)$ to $\varphi_{i, \infty}$ as $k \rightarrow \infty$. Then $\varphi_{i, \infty}$ satisfies

$$
\begin{gathered}
\Delta \varphi_{i, \infty}-m \varphi_{i, \infty}+h^{\prime}(V) \varphi_{i, \infty}=0 \quad \text { in } R_{+}^{N}, \\
\frac{\partial \varphi_{i, \infty}}{\partial y_{n}}=0 \quad \text { in } R^{N-1} \times\{0\}
\end{gathered}
$$

with

$$
\int_{R_{+}^{N}} \varphi_{i, \infty} \frac{\partial V}{\partial y_{j}}=0 \quad j=1, \ldots, N-1 .
$$

From (3.14) we deduce that $\varphi_{i, \infty}$ belongs to the kernel of $S_{0}^{\prime}(V)$ and (3.15) implies that $\varphi_{i, \infty}$ lies in the orthogonal complement of the kernel of $S_{0}^{\prime}(V)$.

Therefore $\varphi_{i, \infty}=0$. As in the proof of Proposition 3.1 we show by the elliptic regularity estimate (3.12) that $\left\|\Phi_{k}\right\|_{H^{2}} \rightarrow 0$ as $k \rightarrow \infty$. This contradicts (3.13) and the proof of Proposition 3.2 is finished.

We are now in a position to solve the equation

$$
\pi_{\epsilon, P} \circ \tilde{S}_{\varepsilon}\left(\sum_{i=1}^{M} P V_{i}+\Phi_{\epsilon, \mathbf{P}}\right)=0 .
$$

Since $\left.L_{\epsilon, \mathbf{P}}\right|_{\mathcal{K}_{\epsilon, \mathbf{P}}^{\perp}}$ is invertible (call the inverse $L_{\epsilon, \mathbf{P}}^{-1}$ ) we can rewrite (3.16) as follows

$$
\begin{gathered}
\Phi=-\left(L_{\epsilon, \mathbf{P}}^{-1} \circ \pi_{\epsilon, \mathbf{P}}\right)\left(\tilde{S}_{\epsilon}\left(\sum_{i=1}^{M} P V_{i}\right)\right) \\
-\left(L_{\epsilon, \mathbf{P}}^{-1} \circ \pi_{\epsilon, \mathbf{P}}\right) N_{\epsilon, \mathbf{P}}(\Phi) \\
\equiv M_{\epsilon, \mathbf{P}}(\Phi)
\end{gathered}
$$

where

$$
N_{\epsilon, \mathbf{P}}(\Phi)=\tilde{S}_{\epsilon}\left(\sum_{i=1}^{M} P V_{i}+\Phi\right)
$$




$$
-\left[\tilde{S}_{\epsilon}\left(\sum_{i=1}^{M} P V_{i}\right)+\tilde{S}_{\epsilon}^{\prime}\left(\sum_{i=1}^{M} P V_{i}\right) \Phi\right]
$$

and the operator $M_{\epsilon, \mathbf{P}}$ is defined by the last equation for $\Phi \in H_{N}^{2}\left(\Omega_{\epsilon}\right)$. We are going to show that the operator $M_{\epsilon, P_{1}, \ldots, P_{M}}$ is a contraction on

$$
B_{\epsilon, \delta} \equiv\left\{\Phi \in H^{2}\left(\Omega_{\epsilon}\right) \mid\|\Phi\|_{H^{2}\left(\Omega_{\epsilon}\right)}<\delta\right\}
$$

if $\delta$ is small enough.

In fact we have the following lemma.

Lemma 3.3. For $\epsilon$ sufficiently small, we have

$$
\begin{aligned}
& \left|N_{\epsilon, \mathbf{P}}\right| \leq C\left(\left|\Phi_{\epsilon, \mathbf{P}}\right|^{1+\sigma}+\left|\Phi_{\epsilon, \mathbf{P}}\right|^{p_{1}}\right) \\
& \left\|S_{\epsilon}\left(\sum_{i=1}^{K} P w_{i}\right)\right\|_{L^{2}\left(\Omega_{\epsilon}\right)} \leq C \epsilon^{\frac{1+\sigma}{2}}
\end{aligned}
$$

Proof: (3.18) follows from the mean value theorem.

On the other hand, (3.19) follows easily by the fact that in the expression $\tilde{S}_{\epsilon}\left(\sum_{i=1}^{K} P V_{i}\right)$ the functions $P V_{i}$ are essentially separated from one another.

We have

$$
\begin{gathered}
\left\|M_{\epsilon, \mathbf{P}}(\Phi)\right\|_{H^{2}\left(\Omega_{\epsilon}\right)} \leq \lambda^{-1}\left(\left\|\pi_{\epsilon, \mathbf{P}} \circ N_{\epsilon, \mathbf{P}}(\Phi)\right\|_{L^{2}\left(\Omega_{\epsilon}\right)}\right. \\
\left.+\left\|\pi_{\epsilon, \mathbf{P}} \circ\left(\tilde{S}_{\epsilon}\left(\sum_{i=1}^{K} P V_{i}\right)\right)\right\|_{L^{2}\left(\Omega_{\epsilon}\right)}\right) \\
\leq \lambda^{-1} C\left(c(\delta) \delta+\epsilon^{(1+\sigma) / 2}\right)
\end{gathered}
$$

where $\lambda>0$ is independent of $\delta>0$ and $c(\delta) \rightarrow 0$ as $\delta \rightarrow 0$. Similarly we show that

$$
\left\|M_{\epsilon, \mathbf{P}}(\Phi)-M_{\epsilon, \mathbf{P}}\left(\Phi^{\prime}\right)\right\|_{H^{2}\left(\Omega_{\epsilon}\right)} \leq \lambda^{-1} C\left(\epsilon^{(1+\sigma) / 2}+c(\delta) \delta\right)\left\|\Phi-\Phi^{\prime}\right\|_{H^{2}\left(\Omega_{\epsilon}\right)}
$$

where $c(\delta) \rightarrow 0$ as $\delta \rightarrow 0$. Therefore $M_{\epsilon, \mathbf{P}}$ is a contraction on $B_{\delta}$. The existence of a fixed point $\Phi_{\epsilon, \mathbf{P}}$ now follows from the Contraction Mapping Principle. Furthermore, $\Phi_{\epsilon, \mathbf{P}}$ is a solution of (3.17).

Because of

$$
\left\|\Phi_{\epsilon, \mathbf{P}}\right\|_{H^{2}\left(\Omega_{\epsilon, \mathbf{P}}\right)} \leq \lambda^{-1}\left(\left\|N_{\epsilon, \mathbf{P}}\left(\Phi_{\epsilon, \mathbf{P}}\right)\right\|_{L^{2}\left(\Omega_{\epsilon}\right)}\right.
$$




$$
\begin{gathered}
\left.+\left\|\tilde{S}_{\epsilon}\left(\sum_{i=1}^{K} P V_{i}\right)\right\|_{L^{2}\left(\Omega_{\epsilon}\right)}\right) \\
\leq \lambda^{-1}\left(c \epsilon^{(1+\sigma) / 2}+c(\delta)\left\|\Phi_{\epsilon, \mathbf{P}}\right\|_{H^{2}\left(\Omega_{\epsilon}\right)}\right)
\end{gathered}
$$

we have

$$
\left\|\Phi_{\epsilon, \mathbf{P}}\right\|_{H^{2}} \leq C \epsilon^{(1+\sigma) / 2} .
$$

We have proved the following lemma.

Lemma 3.4. There exists $\bar{\epsilon}>0$ such that for every $(N+1)$-tuple $\epsilon, P_{1}, \ldots, P_{M}$ with $0<\epsilon<\bar{\epsilon}$ and $P_{i} \in \partial \Omega$ and $\min _{i, j=1, \ldots, M, i \neq j} \operatorname{dist}\left(P_{i}, P_{j}\right) \geq \delta$ there exists a unique $\Phi_{\epsilon, \mathbf{P}} \in \mathcal{K}_{\epsilon, \mathbf{P}}^{\perp}$ satisfying $\tilde{S}_{\epsilon}\left(\sum_{i=1}^{M} P V_{i}+\Phi_{\epsilon, \mathbf{P}}\right) \in \mathcal{C}_{\epsilon, \mathbf{P}}^{\perp}$ and

$$
\left\|\Phi_{\epsilon, \mathbf{P}}\right\|_{H^{2}\left(\Omega_{\epsilon}\right)} \leq C \epsilon^{(1+\sigma) / 2} .
$$

We need another statement about the asymptotic behavior of the function $\Phi_{\epsilon, \mathbf{P}}$ as $\epsilon \rightarrow 0$, which gives an expansion in $\epsilon$ and is stated as follows.

Proposition 3.5. We have

$$
\Phi_{\epsilon, \mathbf{P}}(x)=\epsilon\left(\sum_{i=0}^{M} \Phi_{0}\left(\frac{x-P_{i}}{\varepsilon}\right) \chi\left(x-P_{i}\right)\right)+\epsilon^{2} \Psi_{\epsilon, \mathbf{P}}(x)
$$

where

$$
\left\|\Psi_{\epsilon, \mathbf{P}}\right\|_{\epsilon} \leq C
$$

Here $\Phi_{0}$ is the unique solution of

$$
\begin{gathered}
\Delta \Phi_{0}-m \Phi_{0}+h^{\prime}(V) \Phi_{0}-h^{\prime}(V) v_{1}=0, \quad \text { in } R_{+}^{N}, \\
\frac{\partial \Phi_{0}}{\partial y_{N}}=0 \quad \text { on } \partial R_{+}^{N},
\end{gathered}
$$


where $L_{0}=\Delta-m+h^{\prime}(V), L_{0}: H_{N}^{2}\left(R_{+}^{N}\right) \rightarrow L^{2}\left(R_{+}^{N}\right)$.

Proof. Note that the kernel of $L_{0}$ is

$$
\left\{\frac{\partial V}{\partial y_{j}} \mid j=1, \ldots, N-1\right\} \text {. }
$$

Furthermore we have

$$
\left|\Phi_{0}\right| \leq C \exp (-\mu|y|) \quad \text { for } \mu<\sqrt{m} .
$$

The definitions of $\Omega_{0}, \chi, \rho$ and $T$ are as in section 2. Our strategy is to decompose $\Psi_{\epsilon, \mathbf{P}}$ into three parts and show that each of them is bounded in $\|\cdot\|_{\epsilon}$ as $\epsilon \rightarrow 0$. That means we make the ansatz

$$
\Psi_{\epsilon, \mathbf{P}}(x)=\sum_{i=1}^{M}\left[\Psi_{\epsilon, i}^{1}(x)+\Psi_{\epsilon, i}^{2,1}(x)\right]+\Psi_{\epsilon}^{2,2}(x)
$$

where the functions $\Psi_{\epsilon, i}^{1}, \Psi_{\epsilon, i}^{2,1}, \Psi_{\epsilon, i}^{2,2}$ will be defined as follows. Let $\Psi_{\epsilon, i}^{1}$ be the unique solution of

$$
\begin{gathered}
\epsilon^{2} \Delta \Psi_{\epsilon, i}^{1}-m \Psi_{\epsilon, i}^{1}=0 \quad \text { in } \Omega \\
\frac{\partial \Psi_{\epsilon, i}^{1}}{\partial \nu}=g_{\epsilon, i} \quad \text { on } \partial \Omega
\end{gathered}
$$

where

$$
g_{\epsilon, i}(x)=-\frac{\partial}{\partial \nu_{x}}\left[\Phi_{0}\left(\frac{x-P_{i}}{\varepsilon}\right) \chi\left(x-P_{i}\right)\right] .
$$

Since $\left\|g_{\epsilon, i}\right\|_{L^{2}} \leq C$ there exists a constant $C>0$ such that

$$
\left\|\Psi_{\epsilon, i}^{1}\right\|_{\varepsilon} \leq C
$$

Define $\Psi_{\epsilon, i}^{2,1}$ by

$$
\Psi_{\epsilon, i}^{2,1}(x)=-\frac{1}{\epsilon} \tilde{\pi} \circ \Phi_{0}\left(\frac{x-P_{i}}{\varepsilon}\right) \chi\left(x-P_{i}\right)-\tilde{\pi} \circ \Psi_{\epsilon, i}^{1}(x)
$$

where $\tilde{\pi}$ is the projection of $L^{2}\left(\Omega_{\epsilon}\right)$ onto $\mathcal{K}_{\epsilon, \mathbf{P}}$. Because of the exponential decay of $\Phi_{0}$, the smoothness of $\chi$ and and by (3.24) it follows that

$$
\left\|\Psi_{\epsilon, i}^{2,1}\right\|_{\epsilon} \leq C
$$


Finally, define $\Psi_{\epsilon, i}^{2,2}(x)$ to be the unique solution in $H_{N}^{2}(\Omega)$ of the following equation

$$
\begin{gathered}
\epsilon^{2} \Delta \Psi_{\epsilon}^{2,2}-m \Psi_{\epsilon}^{2,2}+h^{\prime}\left(\sum_{i=1}^{M} P V_{i}\right) \Psi_{\epsilon}^{2,2}=-\frac{1}{\epsilon^{2}} f_{\epsilon} \quad \text { in } \Omega \\
\frac{\partial \Psi_{\epsilon}^{2,2}}{\partial \nu}=0 \quad \text { on } \partial \Omega
\end{gathered}
$$

where

$$
f_{\epsilon}=\tilde{L}_{\epsilon}\left(\Phi_{\epsilon, \mathbf{P}}-\sum_{i=1}^{M}\left[\epsilon \Phi_{0}\left(\left(x-P_{i}\right) / \varepsilon\right) \chi\left(x-P_{i}\right)-\epsilon^{2}\left(\Psi_{\epsilon, i}^{1}+\Psi_{\epsilon, i}^{2,1}\right)\right]\right) .
$$

Note that the right-hand side of the last equation lies in $\mathcal{C}_{\epsilon, \mathbf{P}}^{\perp}$ since

$$
\Phi_{\epsilon, \mathbf{P}}-\sum_{i=1}^{M}\left[\epsilon \Phi_{0}\left(\left(x-P_{i}\right) / \varepsilon\right) \chi\left(x-P_{i}\right)-\epsilon^{2}\left(\Psi_{\epsilon, i}^{1}+\Psi_{\epsilon, i}^{2,1}\right)\right] \in H_{N}^{2} .
$$

This is clear for $\Phi_{\epsilon, \mathbf{P}}$ by definition. By construction we have $-\epsilon \Phi_{0} \chi-\epsilon^{2}\left(\Psi_{\epsilon, i}^{1}+\right.$ $\left.\Psi_{\epsilon, i}^{2,1}\right)$ satisfies the Neumann boundary condition. By (3.22) and the smoothness of $\chi$ we conclude that $\Phi_{0} \chi \in H^{2}$. By (3.23) we deduce that $\Psi_{\epsilon, i}^{1} \in H^{2}$. Finally, since $e_{i j} \in H^{2}$ where

$$
e_{i j}=\frac{\partial V_{i}}{\partial \tau\left(P_{i, j}\right)} /\left\|\frac{\partial V_{i}}{\partial \tau\left(P_{i, j}\right)}\right\|_{L^{2}\left(\Omega_{\epsilon}\right)} \text { for } i=1, \ldots, M, j=1, \ldots, N-1
$$

we have $\Psi_{\epsilon, i}^{2,1} \in H^{2}$. Therefore $f_{\epsilon} \in \mathcal{C}_{\epsilon, \mathbf{P}}^{\perp}$. Furthermore, the following lemma is true.

Lemma 3.6. The function $f_{\epsilon}$ satisfies

$$
\left\|f_{\epsilon}\right\|_{\epsilon} \leq C \epsilon^{2}
$$

Proof. We have

$$
\begin{gathered}
f_{\epsilon}=\tilde{S}_{\varepsilon}\left(\sum_{i=1}^{M} P V_{i}\right)\left(\Phi_{\epsilon}-\sum_{i=1}^{M}\left[\left(\epsilon \Phi_{0}\left(\frac{x-P_{i}}{\varepsilon}\right) \chi\left(x-P_{i}\right)-\epsilon^{2}\left(\Psi_{\epsilon, i}^{1}+\Psi_{\epsilon, i}^{2,1}\right)\right]\right)\right. \\
=-h\left(\sum_{i=1}^{M} P V_{i}\right)+h\left(\sum_{i=1}^{M} V_{i}\right)+\epsilon h^{\prime}\left(\sum_{i=1}^{M} V_{i}\right) v_{1} \chi+N_{\epsilon}^{\prime}\left(\Phi_{\epsilon}\right)
\end{gathered}
$$


where

$$
\begin{gathered}
N_{\epsilon}^{\prime}\left(\Phi_{\varepsilon}\right)=\frac{1}{\left|\Omega_{\varepsilon}\right|} \int_{\Omega_{\varepsilon}} h^{\prime}\left(\sum_{i=1}^{M} P V_{i}\right) \Phi_{\epsilon} \\
+\frac{1}{\left|\Omega_{\varepsilon}\right|} \int_{\Omega_{\varepsilon}}\left[h\left(\sum_{i=1}^{M} P V_{i}\right)-h\left(\sum_{i=1}^{M} V_{i}\right)\right] \\
-\left[h\left(\sum_{i=1}^{M} P V_{i}\right)+\Phi_{\epsilon}\right) \\
\left.-h\left(\sum_{i=1}^{M} P V_{i}\right)-h^{\prime}\left(P V_{i}\right) \Phi_{\epsilon}\right] \\
+\frac{1}{\left|\Omega_{\varepsilon}\right|} \int_{\Omega_{\varepsilon}}\left[h\left(P V_{i}\right)+\Phi_{\epsilon}\right) \\
\left.-h\left(\sum_{i=1}^{M} P V_{i}\right)-h^{\prime}\left(\sum_{i=1}^{M} P V_{i}\right) \Phi_{\epsilon}\right] \\
+\epsilon \sum_{i=1}^{M} \Phi_{0}\left(\frac{x-P_{i}}{\varepsilon}\right)\left[\Delta-m+h^{\prime}\left(\sum_{i=1}^{M} P V_{i}\right)\right] \chi\left(x-P_{i}\right) \\
+\epsilon \sum_{i=1}^{M}<\nabla_{x} \Phi_{0}\left(\left(\cdot-P_{i}\right) / \varepsilon\right), \nabla \Phi(\cdot)> \\
+\epsilon_{i=1}^{2}\left[\Delta-m+h^{\prime}\left(P V_{i}\right)\right] \Psi_{\epsilon}^{2,1} . \\
+\epsilon^{2} h^{\prime}\left(\sum_{i=1}^{M} P V_{i}\right) \Psi_{\epsilon, i}^{1}
\end{gathered}
$$

Note that

$$
\begin{gathered}
\|-h\left(\sum_{i=1}^{M} P V_{i}\right)+h\left(\sum_{i=1}^{M} V_{i}\right) \\
+\epsilon h^{\prime}\left(\sum_{i=1}^{M} V_{i}\right) v_{1} \chi\left(x-P_{i}\right) \|_{L^{2}} \\
\leq\left\|-h\left(\sum_{i=1}^{M} P V_{i}\right)+h\left(\sum_{i=1}^{M} V_{i}\right)+\epsilon h^{\prime}\left(\sum_{i=1}^{M} V_{i}\right) v_{1}\right\|_{L^{2}} \\
+\left\|\epsilon\left(-h^{\prime}\left(\sum_{i=1}^{M} V_{i}\right) v_{1}+h^{\prime}\left(\sum_{i=1}^{M} V_{i}\right) v_{1} \chi\right)\right\|_{L^{2}} \\
\leq C\left(\epsilon^{2}+\exp \left(-\mu R_{0}\right)\right)
\end{gathered}
$$

by the definition of $\chi$ and the exponential decay of $V$. Furthermore

$$
\left\|N_{\epsilon, P_{i}}^{\prime}(\Phi)\right\|_{L^{2}} \leq C \epsilon^{2} .
$$


This proves Lemma 3.6. $\square$

By Lemma 3.6 and the invertibility of

$$
\tilde{L}_{\epsilon}: H_{N}^{2} \cap \mathcal{K}_{\epsilon, \mathbf{P}}^{\perp} \rightarrow \mathcal{C}_{\epsilon, \mathbf{P}}^{\perp}
$$

Proposition 3.5 follows.

\section{The Reduced PRoblem}

In this section we solve the reduced problem and prove our main theorem. By Lemma 3.3 there exists a unique solution $\Phi_{\epsilon, \mathbf{P}} \in \mathcal{K}_{\epsilon, \mathbf{P}}^{\perp}$ such that the function

$$
u_{\varepsilon}=\sum_{i=1}^{M} P V_{i}+\Phi_{\epsilon, \mathbf{P}}
$$

satisfies

$$
\begin{gathered}
S_{\epsilon}\left(u_{\epsilon}\right)=S_{\epsilon}\left(\sum_{i=1}^{M} P V_{i}+\Phi_{\epsilon, \mathbf{P}}\right) \\
=\epsilon^{2} \Delta u_{\epsilon}-m u_{\epsilon}+h\left(u_{\epsilon}\right)-\frac{1}{|\Omega|} \int_{\Omega} h\left(u_{\epsilon}\right) \in \mathcal{C}_{\epsilon, \mathbf{P}}^{\perp}
\end{gathered}
$$

Our idea is to find $\mathbf{P}=\left(P_{1}, \ldots, P_{M}\right)$ with $P_{i} \in \partial \Omega$ pairwise different such that

$$
S_{\epsilon}\left(u_{\epsilon}\right) \perp \mathcal{C}_{\epsilon, \mathbf{P}}
$$

Let

$$
W_{\epsilon, j}(P)=\frac{1}{\epsilon^{N+1}} \int_{\Omega}\left(S_{\epsilon}\left(u_{\epsilon}\right) \frac{\partial P_{\Omega_{\epsilon, P}} V}{\partial \tau_{P_{j}}}\right)
$$

and

$$
W_{\varepsilon}(P)=\left(W_{\varepsilon, 1}(P), \ldots, W_{\varepsilon, N-1}(P)\right) .
$$

Then $W_{\epsilon}(P)$ is a continuous map of $P$. We want to find $\mathbf{P}=\left(P_{1}, \ldots, P_{M}\right)$ such that

$$
W_{\varepsilon}\left(P_{i}\right)=0 \quad \text { for } j=1, \ldots, M .
$$

Let us now calculate $W_{\varepsilon}\left(P_{i}\right)$.

First of all, from the conditions on $h$, we have

$$
\int_{\Omega} h\left(u_{\epsilon}\right) \leq C \epsilon^{N}
$$


Hence by Proposition 2.2 we deduce that

$$
\begin{gathered}
\frac{1}{\epsilon^{N+1}} \int_{\Omega}\left(\int_{\Omega} h\left(u_{\epsilon}\right)\right) \frac{\partial P_{\Omega_{\epsilon, P}} V}{\partial \tau_{P_{j}}}=\int_{\Omega} h\left(u_{\epsilon}\right) \frac{1}{\epsilon^{N+1}} \int_{\Omega} \frac{\partial P_{\Omega_{\epsilon, P}} V}{\partial \tau_{P_{j}}} \\
=\mathcal{O}\left(\epsilon^{N}\right)\left(\frac{1}{\epsilon^{N+1}} \int_{\Omega}\left(\frac{\partial V((\cdot-P) / \varepsilon)}{\partial P_{j}}+w_{1}(y) \chi(x-P)+\epsilon w_{2, i}^{\epsilon}(x)\right)\right) \\
=\mathcal{O}\left(\epsilon^{N}\right) \frac{1}{\epsilon^{N+1}}\left[\begin{array}{l}
\left.\mathcal{O}(\exp (-\sigma / \epsilon))+\epsilon \int_{\Omega} w_{2, i}^{\epsilon}\right] \\
=\mathcal{O}\left(\epsilon^{N / 2}\right)
\end{array}\right.
\end{gathered}
$$

because

$$
\frac{1}{\epsilon^{N}} \int_{\Omega} w_{2, i}^{\epsilon} \leq \frac{1}{\epsilon^{N / 2}}\left\|w_{2, i}^{\epsilon}\right\|_{L^{2}(\Omega)}
$$

and by Proposition 2.3. On the other hand, since

we conclude that

$$
\begin{gathered}
\epsilon^{2} \Delta \frac{\partial P_{\Omega_{\epsilon, P}} V}{\partial \tau_{P_{j}}}-m \frac{\partial P_{\Omega_{\epsilon, P}} V}{\partial \tau_{P_{j}}} \\
+h^{\prime}(V) \frac{\partial V}{\partial y_{j}}=0
\end{gathered}
$$

$$
\begin{gathered}
\int_{\Omega}\left[\epsilon^{2} \Delta u_{\epsilon}-m u_{\epsilon}+h\left(u_{\epsilon}\right)\right] \frac{\partial P_{\Omega_{\epsilon, P}} V}{\partial \tau_{P_{j}}} \\
=\int_{\Omega}\left\{h\left(u_{\epsilon}\right) \frac{\partial P_{\Omega_{\epsilon, P}} V}{\partial \tau_{P_{j}}}\right. \\
\left.+\left[\epsilon^{2} \Delta \frac{\partial P_{\Omega_{\epsilon, P}} V}{\partial \tau_{P_{j}}}-m \frac{\partial P_{\Omega_{\epsilon, P}} V}{\partial \tau_{P_{j}}}\right] u_{\epsilon}\right\} \\
=\int_{\Omega}\left[h\left(u_{\epsilon}\right) \frac{\partial P_{\Omega_{\epsilon, P}} V}{\partial \tau_{P_{j}}}-h^{\prime}(V) \frac{\partial V}{\partial P_{j}} u_{\epsilon}\right] \\
=\int_{\Omega}\left[h\left(\sum_{i=1}^{M} P V_{i}\right)+\Phi_{\epsilon, \mathbf{P}}\right)-h\left(\sum_{i=1}^{M} P V_{i}\right) \\
+\int_{\Omega}\left[h^{\prime}\left(\sum_{i=1}^{M} P V_{i}\right) \Phi_{\epsilon, \mathbf{P}}\right] \frac{\partial P_{\Omega, P} V}{\partial \tau_{P_{j}}} \\
+\int_{\Omega}\left[h\left(\sum_{i=1}^{M} P V_{i}\right) \frac{\partial P_{\Omega_{\epsilon, P}} V}{\partial \tau_{P_{j}}}-h^{\prime}(V) \frac{\partial V((x-P) / \varepsilon)}{\partial P_{j}}\right] \\
\times \Phi_{\epsilon, \mathbf{P}} \\
=I_{\epsilon}^{1}+I_{\epsilon}^{2}+J_{\epsilon}
\end{gathered}
$$


where $I_{\epsilon}^{1}, I_{\epsilon}^{2}$, and $J_{\epsilon}$ are defined by the last equality. We first calculate $I_{\epsilon}^{2}$.

$$
\begin{aligned}
I_{\epsilon}^{2}= & \int_{\Omega}\left[h^{\prime}\left(\sum_{i=1}^{M} P V_{i}\right) \frac{\left.\partial P_{\Omega_{\epsilon}} V(x-P) / \varepsilon\right)}{\partial \tau_{P_{j}}}-h^{\prime}(V) \frac{\partial V(x-P) / \varepsilon)}{\partial \tau_{P_{j}}}\right] \\
& =\int_{\Omega}\left[h^{\prime}\left(\sum_{i=1}^{M} P \Phi_{0}(y) \chi(x-P)+\epsilon^{2} \Psi_{\epsilon}(x)\right) d x\right. \\
& +\epsilon^{2} \int_{\Omega}\left[h^{\prime}\left(\sum_{i=1}^{M} P V_{i}\right) \frac{\left.\partial P_{\Omega_{\epsilon}} V(x-P) / \varepsilon\right)}{\partial \tau_{P_{j}}}-h^{\prime}(V) \frac{\partial V}{\partial \tau_{P_{j}}}\right] \epsilon \Phi_{0} \chi \\
& =\epsilon I_{\epsilon}^{2,1}+\epsilon_{P_{j}} I_{\epsilon}^{2,2} .
\end{aligned}
$$

Now assume that $P=P_{i}$. Furthermore, note that

$$
\begin{gathered}
h^{\prime}\left(\sum_{i=1}^{M} P V_{i}\right) \frac{\left.\partial P_{\Omega_{\epsilon}} V(x-P) / \varepsilon\right)}{\partial \tau_{P_{j}}}-h^{\prime}(V) \frac{\partial V}{\partial \tau_{P_{j}}} \\
=\left[h^{\prime}\left(\sum_{i=1}^{M} P V_{i}\right)-h^{\prime}(V)\right] \frac{\left.\partial P_{\Omega_{\epsilon}} V(x-P) / \varepsilon\right)}{\partial \tau_{P_{j}}} \\
\quad+h^{\prime}(V)\left[\frac{\left.\partial P_{\Omega_{\epsilon}} V(x-P) / \varepsilon\right)}{\partial \tau_{P_{j}}}-\frac{\partial V}{\partial \tau_{P_{j}}}\right]
\end{gathered}
$$

and

$$
\begin{gathered}
\int_{\Omega}\left[h^{\prime}\left(\sum_{i=1}^{M} P V_{i}\right)-h^{\prime}(V)\right] \frac{\left.\partial P_{\Omega_{\epsilon}} V(x-P) / \varepsilon\right)}{\partial \tau_{P_{j}}} \Phi_{0} \chi(x-P) \\
=\int_{\Omega} h^{\prime \prime}(V)\left(\sum_{i=1}^{M} P V_{i}-V\right) \frac{\partial P_{\Omega_{\epsilon, P}} V}{\partial \tau_{P_{j}}} \Phi_{0} \chi(x-P) \\
\left.+\int_{\Omega} h^{\prime \prime \prime}\left(v_{1}\right)\left(\sum_{i=1}^{M} P V_{i}-V(x-P) / \varepsilon\right)\right)^{2} \frac{\left.\partial P_{\Omega_{\epsilon}} V(x-P) / \varepsilon\right)}{\partial \tau_{P_{j}}} \Phi_{0} \chi(x-P) \\
+\mathcal{O}(\exp (-\delta / \epsilon)) \\
=\mathcal{O}\left(\epsilon^{N+1}\right)
\end{gathered}
$$

since $\Phi_{0}$ is even and $\left(V-\sum_{i=1}^{M} P V_{i}\right) \chi\left(x-P_{i}\right)=\epsilon v_{1}+O\left(\varepsilon^{2}\right)$ where $v_{1}$ is even.

By Proposition 2.1 we have

$$
\int\left|\Psi_{\epsilon}\right|^{2} \leq C \epsilon^{N}
$$

Hence we conclude that

$$
\left|I_{\epsilon}^{2,2}\right| \leq \mathcal{O}\left(\epsilon^{N}\right)
$$

and

$$
\left|I_{\epsilon}^{2}\right| \leq \mathcal{O}\left(\epsilon^{N+2}\right) .
$$


We next compute $I_{\epsilon}^{1}$, again assuming that $P=P_{i}$,

$$
\begin{gathered}
I_{\epsilon}^{1}=\int_{\Omega} h^{\prime \prime}\left(\sum_{i=1}^{M} P V_{i}\right) \Phi_{\epsilon, P}^{2} \frac{\partial P_{\Omega_{\epsilon, P}} V}{\partial \tau_{P_{j}}} \\
+\int_{\Omega} h^{\prime \prime \prime}\left(v_{1}+\sum_{i=1}^{M} P V_{i}\right) \Phi_{\epsilon, P}^{3} \frac{\left.\partial P_{\Omega_{\epsilon}} V(x-P) / \varepsilon\right)}{\partial \tau_{P_{j}}} \\
=\int_{\Omega} h^{\prime \prime}\left(\sum_{i=1}^{M} P V_{i}\right) \epsilon^{2}\left[\Phi_{0}^{2} \chi^{2}+2 \epsilon \Phi_{0} \chi \Psi_{\epsilon, P}+\epsilon^{2} \Psi_{\epsilon, P}^{2}\right] \frac{\left.\partial P_{\Omega_{\epsilon}} V(x-P) / \varepsilon\right)}{\partial \tau_{P_{j}}} \\
+\mathcal{O}\left(\epsilon^{N+2}\right) \\
=\mathcal{O}\left(\epsilon^{N+2}\right)
\end{gathered}
$$

since $\Phi_{0}$ is even. Finally, we compute the term $J_{\epsilon}$.

$$
\begin{gathered}
J_{\epsilon}=\int_{\Omega}\left[h\left(\sum_{i=1}^{M} P V_{i}\right)-h(V)\right] \frac{\partial P V_{i}}{\partial \tau_{P_{j}}} \\
=\int_{\Omega} h^{\prime}(V)\left(\sum_{i=1}^{M} P V_{i}-V\right) \frac{\left.\partial P_{\Omega_{\epsilon}} V(x-P) / \varepsilon\right)}{\partial \tau_{P_{j}}} \\
+h^{\prime \prime}(V)\left(\sum_{i=1}^{M} P V_{i}-V\right)^{2} \frac{\left.\partial P_{\Omega_{\epsilon}} V(x-P) / \varepsilon\right)}{\partial \tau_{P_{j}}}+\mathcal{O}\left(\epsilon^{N+2}\right) \\
=\epsilon \int_{\Omega} h^{\prime}(V)\left(v_{1} \chi+\epsilon\left(v_{2} \chi+v_{3} \chi\right)+\epsilon^{2} \Psi_{\epsilon}\right)\left(\frac{\partial V}{\partial P_{j}}+w_{1}+\epsilon w_{2}^{\epsilon}(x)\right) \\
+\epsilon^{2} \int_{\Omega} h^{\prime \prime}(V)\left(v_{1}^{2} \chi^{2}+\epsilon\left(\Psi_{\epsilon}^{1}\right)^{2}\right) \frac{\left.\partial P_{\Omega_{\epsilon}} V(x-P) / \varepsilon\right)}{\partial \tau_{P_{j}}}+\mathcal{O}\left(\epsilon^{N+2}\right) \\
=\epsilon^{2} \int_{\Omega} h^{\prime}(V) v_{3} \frac{\partial V}{\partial P_{j}}+\mathcal{O}\left(\epsilon^{N+2}\right) \\
=-\epsilon^{N+1}\left(\int_{\Omega_{\epsilon, P}} h^{\prime}(V) v_{3} \frac{\partial V}{\partial y_{j}}\right)+\mathcal{O}\left(\epsilon^{N+2}\right) \\
=-\epsilon^{N+1} \int_{R_{+}^{N}} h^{\prime}(V) v_{3} \frac{\partial V}{\partial y_{j}}+\mathcal{O}\left(\epsilon^{N+2}\right) .
\end{gathered}
$$

We also have

$$
\begin{gathered}
\int_{R_{+}^{N}} h^{\prime}(V) v_{3} \frac{\partial V}{\partial y_{j}}=-\int_{R_{+}^{N}}\left(\Delta \frac{\partial V}{\partial y_{j}}-m \frac{\partial V}{\partial y_{j}}\right) v_{3} \\
=\int_{\partial R_{+}^{N}} \frac{\partial v_{3}}{\partial y_{N}} \frac{\partial V}{\partial y_{j}}-v_{3} \frac{\partial}{\partial y_{N}} \frac{\partial V}{\partial y_{j}} \\
=-\frac{1}{3} \int_{R^{N-1}}\left(\frac{V^{\prime}}{|y|}\right)^{2} \sum_{k, l, m=1}^{N-1} \rho_{k l m}(0) y_{k} y_{l} y_{m} y_{j} d y
\end{gathered}
$$




$$
\begin{gathered}
=-\frac{1}{3} \int_{R^{N-1}}\left(\frac{V^{\prime}}{|y|}\right)^{2} \sum_{k, l, m=1}^{N-1} y_{k} y_{l} y_{m} y_{j} \rho_{k l m}(0) d y \\
=-\frac{1}{3} \int_{R^{N-1}}\left(\frac{V^{\prime}}{|y|}\right)^{2} y_{j}^{2} \sum_{l, m=1}^{N-1} y_{l} y_{m} \rho_{j l m}(0) d y \\
=\nu \rho_{j k k}(0) \\
=\nu \nabla_{j} H(P)
\end{gathered}
$$

where

$$
\nu=-\sum_{k=1}^{N-1} \frac{1}{3} \int_{R^{N-1}}\left(\frac{V^{\prime}}{|y|}\right)^{2} y_{j}^{2} y_{k}^{2} d y \neq 0 .
$$

Combining $I_{\epsilon}^{1}, I_{\epsilon}^{2}, J_{\epsilon}$, we obtain

$$
W_{\epsilon}(P)=\nu \nabla_{\tau_{P_{0}}} H(P)+W_{\epsilon}^{\prime}(P)
$$

where $W_{\epsilon}^{\prime}(P)$ is continuous in $P$ and $W_{\epsilon}^{\prime}(P)=\mathcal{O}(\epsilon)$ uniformly in $P$. Suppose that at $P_{0, i}$, we have $\operatorname{det}\left(\nabla_{\tau P_{0, i}} \nabla_{\tau P_{0, i}} H\left(P_{0, i}\right)\right) \neq 0$ then the standard Brouwer fixed point theorem shows that for $\epsilon<<1$ there exist $P_{\epsilon, i}$ such that $W_{\epsilon}\left(P_{\epsilon, i}\right)=0$ and $P_{\epsilon, i} \rightarrow P_{0, i}$ for $i=1,2, \ldots, M$.

Thus we have proved the following proposition.

Proposition 4.1. For $\epsilon$ sufficiently small there exist points $P_{\epsilon, 1}, P_{\varepsilon, 2}, \ldots, P_{\varepsilon, M}$ with $P_{\epsilon, i} \rightarrow P_{0, i}$ such that $W_{\epsilon}\left(P_{\epsilon, i}\right)=0$.

By Lemma 3.3 and Proposition 4.1 we have

$$
S_{\epsilon}\left(v_{\epsilon}\right)=0
$$

which is the same as to say

$$
\begin{gathered}
\epsilon^{2} \Delta v_{\epsilon}-m v_{\epsilon}+h\left(u_{\epsilon}\right)-\frac{1}{|\Omega|} \int_{\Omega} h\left(v_{\epsilon}\right)=0 \quad \text { in } \Omega, \\
\frac{\partial v_{\epsilon}}{\partial \nu}=0 \quad \text { on } \partial \Omega .
\end{gathered}
$$

Hence $\int_{\Omega} v_{\epsilon}=0$. Let $u_{\epsilon}=\bar{m}-v_{\epsilon}$. We have

$$
\begin{gathered}
\epsilon^{2} \Delta u_{\epsilon}-f\left(u_{\epsilon}\right)=\sigma_{\epsilon}, \\
\partial u_{\epsilon} / \partial \nu=0 \quad \text { on } \partial \Omega
\end{gathered}
$$




$$
\int_{\Omega} u_{\epsilon}=\bar{m}|\Omega|
$$

meaning that $u_{\epsilon}$ is a solution of the Cahn-Hilliard equation. Moreover, we have

$$
\left\|v_{\epsilon}-\sum_{i=1}^{M} V\left(\frac{x-P_{\epsilon, i}}{\epsilon}\right)\right\|_{\epsilon} \rightarrow 0
$$

and $P_{\epsilon, i} \rightarrow P_{0, i} \in \partial \Omega$.

Finally, we study the shape of the solutions $v_{\epsilon}$. Let $P_{\epsilon}$ be any local maximum point of $v_{\epsilon}$. Then from (1.2) we deduce that

$$
m v_{\epsilon}-h\left(v_{\epsilon}\right)+\frac{1}{|\Omega|} \int_{\Omega} h\left(v_{\epsilon}\right) \leq 0 .
$$

But $\epsilon^{-N} \int_{\Omega} h\left(v_{\epsilon}\right) \rightarrow M \int_{R_{+}^{N}} h(V)>0$, hence

$$
m v_{\epsilon}-h\left(v_{\epsilon}\right)<0 \text {. }
$$

So $v_{\epsilon}\left(P_{\epsilon}\right) \geq a_{1}>0$. On the other hand, from our construction, we see that

$$
\left\|v_{\epsilon}\right\|_{\epsilon}^{2} \rightarrow \frac{M}{2}\left(\int_{R^{N}}|\nabla V|^{2}+m V^{2}\right) .
$$

By a proof similar to that of Theorem 1.2 in [25], we conclude that $P_{\epsilon, i} \in \partial \Omega$ and there are exactly $M$ such points $P_{\epsilon, i}$.

\section{REFERENCES}

[1] S. Agmon, Lectures on Elliptic Boundary Value Problems, (Van Nostrand, Princeton, 1965).

[2] N. Alikakos, P.W. Bates and X. Chen, 'Convergence of the Cahn-Hilliard equation to the Hele-Shaw model', Arch. Rat. Mech. Anal. 128 (1994) 165-205.

[3] N. Alikakos, P.W. Bates and G. Fusco, 'Slow motion for the Cahn-Hilliard equation in one space dimension', J. Diff. Eqns. 90 (1991) 81-134.

[4] D.G. Aronson and H.F. Weinberger, 'Multidimensional nonlinear diffusion arising in population genetics', Adv. in Math. 30 (1978) 33-76. 
[5] P.W. Bates And P.C. Fife, 'The dynamics of nucleation for the Cahn-Hilliard equation', SIAM J. Appl. Math. 53 (1993) 990-1008.

[6] J.W. Cahn and J.E. Hilliard, 'Free energy of a nonuniform system, I. Interfacial free energy', J. Chem. Phys. 28 (1958) 258-267.

[7] X. Chen and M. Kowalczyk, 'Existence of equilibria for the Cahn-Hilliard equation via local minimizers of the perimeter', Comm. PDE, 21 (1996) 1207-1233.

[8] E.N. DANCER, 'A note on asymptotic uniqueness for some nonlinearities which change sign', Rocky Mountain Math. J., to appear.

[9] C.M. Elliot And D.A. French, 'Numerical studies of the Cahn-Hilliard equation for phase separation', IMA J. Appl. Math. 38 (1987) 97-128.

[10] C.M. Elliot and S. Zheng, 'On the Cahn-Hilliard equation', Arch. Rat. Mech. Anal. 96 (1986) 339-357.

[11] A. Floer And A. Weinstein, 'Nonspreading wave packets for the cubic Schrödinger equation with a bounded potential', J. Funct. Anal. 69 (1986) 397-408.

[12] C. Gui, 'Multi-peak solutions for a semilinear Neumann problem', Duke Math. J. $84(1996)$ 739-769.

[13] B. Gidas, W.-M. Ni And L. Nirenberg, 'Symmetry of positive solutions of nonlinear elliptic equations in $R^{n}$, Mathematical Analysis and Applications, Part A, Adv. Math. Suppl. Studies Vol. 7A, pp. 369-402, (Academic Press, New York, 1981).

[14] R. Gardner and L.A. Peletier, 'The set of positive solutions of semilinear equations in large balls', Proc. Roy. Soc. Edinburgh A 104 (1986) 53-72.

[15] D. Gilbarg and N.S. Trudinger, Elliptic Partial Differential Equations of Second Order, 2nd edition, (Springer, 1983). 
[16] M. Grinfeld And A. Novick-Cohen, 'Counting stationary solutions of the CahnHilliard equation by transversality arguments', Proc. Roy. Soc. Edinburgh Sect. A 125 (1995) 351-370.

[17] M. Grinfeld And A. Novick-Cohn, 'The viscous Cahn-Hilliard equation: Morse decomposition and structure of the global attractor', submitted.

[18] B. Helffer And J. SJÖstrand, 'Multiple wells in the semi-classical limit I', Comm. PDE 9 (1984) 337-408.

[19] J. JANG, 'On spike solutions of singularly perturbed semilinear Dirichlet problems', J. Diff. Eqns 114 (1994) 370-395.

[20] R.V. Kohn And P. Sternberg, 'Local minimizers and singular perturbations', Proc. Roy. Soc. Edinburgh Sect. A 111 (1989) 69-84.

[21] C. Lin, W.-M. Ni AND I. TAKAGI, 'Large amplitude stationary solutions to a chemotaxis system', J. Diff. Eqns. 72 (1988) 1-27.

[22] J.L. Lions And E. Magenes, Non-Homogeneous Boundary Value Problems and Applications, Vol I, (Springer, 1972).

[23] L. ModICA, 'The gradient theory of phase transitions and the minimal interface criterion', Arch. Rational Mech. Anal. 107 (1989) 71-83.

[24] W.-M. Ni, X. PAn And I. TAKAgI, 'Singular behavior of least-energy solutions of a semilinear Neumann problem involving critical Sobolev exponents', Duke Math. J. 67 (1992) 1-20.

[25] W.-M. Ni AND I. TAKAGI, 'On the shape of least energy solutions to a semilinear Neumann problem', Comm. Pure Appl. Math. 41 (1991) 819-851.

[26] W.-M. Ni AND I. TAKAGI, 'Locating the peaks of least energy solutions to a semilinear Neumann problem', Duke Math. J. 70 (1993) 247-281. 
[27] W.-M. Ni AND J. WeI, 'On the location and profile of spike-layer solutions to singularly perturbed semilinear Dirichlet problems', Comm. Pure Appl. Math. 48 (1995) 731-768.

[28] Y.G. OH, 'Existence of semi-classical bound states of nonlinear Schrödinger equations with potentials of the class $(V)_{a}{ }^{\prime}$, Comm. PDE 13(12) (1988) 1499-1519.

[29] Y.G. Он, 'On positive multi-lump bound states of nonlinear Schrödinger equations under multiple-well potentials', Comm. Math. Phys. 131 (1990) 223-253.

[30] R.L. Pego, 'Front migration in the nonlinear Cahn-Hilliard equation', Proc. Roy. Soc. London A 422 (1989) 261-278.

[31] L.A. Peletier ANd J. Serrin, 'Uniqueness of positive solutions of semilinear equations in $R^{n}$, Arch. Rational Mech. Anal. 81 (1983) 181-197.

[32] J. Smoller And A. Wasserman, 'Global bifurcation of steady-state solutions', J. Diff. Eqns 39 (1981) 269-290.

[33] J. WEI, 'On the boundary spike layer solutions of singularly perturbed semilinear Neumann problem', J. Diff. Eqns. 134 (1997)

[34] J. Wei AND M. Winter, 'Stationary solutions for the Cahn-Hilliard equation', Ann. Inst. H. Poincaré Anal. Non Linéaire (1997) to appear.

[35] Zeidler, E., Nonlinear Functional Analysis and its Applications I, Fixed-Point Theorems, (Springer, 1986).

Department of Mathematics, The Chinese University of Hong Kong, Shatin, HONG KONG

Mathematisches Institut A, Universität Stuttgart, D-70511 Stuttgart, GERMANY 